\title{
Violencia en las parejas adolescentes. Implicaciones del sexismo y la religión
}

\section{Teen dating violence. Implications of sexism and religion}

\author{
David Rodríguez Sola ${ }^{1}$ y Encarnación Soriano Ayala ${ }^{2}$
${ }^{1}$ Graduado en Enfermería por la Universidad de Almería. Master en Ciencias de la Sexología. Master en Urgencias y Emergencias Sanitarias. Master en Farmacoterapia. Enfermero del
Hospital de Poniente (Almería-España). E-mail: rodriguezsola@hotmail.com
Directora del grupo de investigación HUM665 "Investigación y evaluación en Educación Intercultural”. E-mail: esoriano@ual.es \\ ${ }^{2}$ Doctora en Ciencias de la Educación. Profesora Titular de la Universidad de Almería (España).
}

\begin{abstract}
Esta investigación ha sido financiada por el proyecto RTI2018-101668-B-I00 Violencia en la pareja adolescente (Teen Dating Violence). Investigación transcultural para la prevención e intervención en contextos socioeducativos.

Ministerio de Ciencia, Innovación y Universidades de España.
\end{abstract}

Universidad de Almería.

España.

\section{Resumen}

La violencia en las parejas adolescentes es un tema de especial importancia por la implicación en la salud y en las relaciones afectivas de los adolescentes. El propósito de este estudio fue determinar la influencia del género y de la religión en la violencia física, de comunicación/relación y psicológica, recibidas por los adolescentes, y en la satisfacción con la vida que estos presentan, además, del papel que juegan las conductas sexistas en esta violencia. Los participantes han sido 1036 adolescentes pertenecientes a siete centros de educación secundaria del sudeste español, de edades comprendidas entre 14 y 19 años, de los que el $48.1 \%$ eran hombres y el $51.9 \%$, mujeres. Para alcanzar los objetivos de la investigación, junto con los datos demográficos y personales, se aplicó la Escala de Satisfacción con la Vida (SWLS), la Escala de Violencia en la pareja desarrollada y validada para este estudio que recoge tres subescalas: violencia psicológica, violencia física, y violencia en la comunicación y relación con otros. Finalmente, el cuestionario sobre conductas sexistas da información sobre creencias falsas y conductas sexistas.

Los resultados muestran niveles más altos de violencia física, psicológica y de comunicación/relación sufrida por los hombres. Aunque son los hombres los que se sienten más satisfechos con la vida y los que manifiestan más conductas sexistas. La creencia religiosa no interviene en la violencia ejercida, pero son los adolescentes practicantes los que se muestran más satisfechos con la vida.

Palabras clave: violencia pareja adolescente, violencia física, psicológica, comunicación/ relación, satisfacción con la vida, conductas sexistas, religión.

\section{Abstract}

Teen dating violence is defined as the physical, sexual, psycho-emotional violence 
that occurs within couple relationships during adolescence. These are relationships that threaten the well-being, health or integrity of the couple and that use mechanisms of control or domination of the couple through coactive or coercive tactics.

With this research we have tried to find out about life satisfaction, the physical, psychological and communicative violence suffered by adolescent men and women who are religious or not. In addition, detect sexist behaviours in young people of both sexes and assess behaviours based on religious practice.

A descriptive, cross-sectional and predictive study was carried out. Cluster random sampling was carried out when selecting secondary schools in South-eastern Spain. The study included 1036 adolescents with ages between 14 and 19 years old, of whom $48.1 \%$ were men and $51.9 \%$ women.

To fulfill the objectives together with demographic and personal data, the Satisfaction with Life Scale (SWLS) is applied, which assesses the cognitive aspects of well-being. The Psychological Violence Scale is applied with the objective of assessing the psychological and emotional violence. To know the physical violence and authoritarian behaviour, the Physical Violence Scale is applied. Finally, the Violence in Communication and Relationship with Others Scale is introduced for studying online violence and relationship violence. The three violence scales have been adapted, developed and validated by Soriano and Aguilera (2017). Finally, the questionnaire on sexist behaviours collects information on false beliefs and sexist behaviours.

The data collection procedure followed the standards set out in the Helsinki Convention (2013) for scientific research involving human participants.

Regarding the results, in the first of the logistic regression models, it is good for boys to go out with many girls, but not the other way around $($ Wald $=14.461 ; \mathrm{p}=.000 ; \mathrm{OR}=$ $4.047)$, it is observed to be that boys are 4.047 times more likely to agree with this attitude than girls. Also, it is statistically significant that sometimes you have to threaten others to know who is in charge, (Wald $=8.107 ; \mathrm{p}=$ .004 ; OR $=2.173$ ), which indicates that the boys are 2.173 times more likely in accordance with that conduct that the girls. It is also statistically significant that when a woman is assaulted by her partner she will have done something to provoke him, (Wald = 16.315; $\mathrm{p}=.000 ; \mathrm{OR}=3.538)$, making it 3.538 times more likely that boys support this attitude than girls. At the same time, it is statistically significant that the violence that occurs within the home is a family matter and should not leave there, $($ Wald $=4.132 ; \mathrm{p}=.042 ; \mathrm{OR}=1.694)$, which also indicates that boys are 1.694 times more likely to adopt this behaviour than girls. Regarding the perception of violence, it is observed that men perceive more violence than women, being significant for the three types of violence studied $(p<.05)$ : Communication, psychological and physical. Personal satisfaction also shows significant results ( $Z$ $=-2.61 ; p=.009)$. In this case, men perceive greatersatisfaction with their life than women.

For students with religious beliefs and practices, statistically significant results were evident only in personal satisfaction $(Z=$ $-3.42 ; \mathrm{p}=.001)$, showing that students who practice a religion have more satisfaction with life.

The results show higher levels of physical, psychological and communication / relationship violence suffered by men. However, men feel more satisfation with life and show more sexist behaviour. Religious belief does not intervene in violence, but the practicing adolescents are those most satisfied with life. Keywords: Teen dating violence, physical, psychological and communication violence, life satisfaction, sexist behaviour, religion.

\section{Introducción}

La violencia en la pareja adolescente (VPA) o teen dating violence (en inglés) se define como la violencia física, sexual, psicoemo- 
cional que se da en el seno de las relaciones de pareja durante la adolescencia (Centers for Disease Control and Prevention, 2012; Children's Safety Network, 2012; Rey-Anacona, 2009; Sánchez, Ortega, Ortega y Viejo, 2008). Se trata de relaciones que atentan contra el bienestar, la salud o la integridad de la pareja (Cornelius y Resseguie, 2007; Lavoie, Robitaille y Hébert, 2000) y que recurren a mecanismos de control o dominio mediante tácticas coactivas o coercitivas (Rubio-Garay, Carrasco, Amor y López-González, 2015).

Las consecuencias de la violencia en el noviazgo entre adolescentes incluyen problemas de salud mental, bajo rendimiento académico, manejo agresivo de conflictos, desajuste psicosocial, problemas de humor, menor satisfacción con la vida, depresión e ideación suicida (Offenhauer y Buchalter, 2011; Penado y Rodicio-García, 2017; Zweig, Lachman, Yahner y Dank, 2014). Las chicas y los chicos que experimentan violencia en el noviazgo durante la adolescencia tienen más probabilidades de ser consumidores de alcohol y otras drogas (Eaton, Davis, Barrios, Brener y Noonan, 2007).

Los estudios indican que la violencia en la adolescencia puede predecir la violencia en la vida marital adulta (Murphy y O’Leary, 1989; O’Leary, Malone y Tyree, 1994; Roscoe y Bensake, 1985; Smith, White y Holland, 2003).

\section{Violencia física, psicológica, en la comunicación y satisfacción con la vida}

Desde la década de 1980, la violencia en la pareja adolescente se considera un problema de salud pública (Valdivia y González, 2014). Un estudio llevado a cabo en España con 11020 chicos y chicas por el Observatorio contra la Violencia de Género y el Ministerio de Igualdad entre 2008 y 2011 comprobó que el $32 \%$ de los adolescentes desarrollaban comportamientos de maltratador y el $23 \%$ habían sufrido violencia física en su relación de pareja (Casabona y Tellado, 2012; Sebas- tián, Verdugo y Ortiz, 2014).

La violencia en las relaciones de pareja adolescente puede adoptar diferentes formas y modos: abusos verbales y emocionales, conductas dominantes y de control, maltrato físico, maltrato psicológico y abuso sexual (Cutbush, Williams, Miller, Gibbs y Clinton-Sherrod, 2018; Herranz Bellido, 2013; Perles, San Martin y Canto, 2019; Rivas, Roldan, Gimeno y Díaz, 2015; Yan, Howard, Beck, Shattuck y Hallmark-Kerr, 2009). Los problemas de las parejas adolescentes tienen su inicio en la primera fase de la relación; es cuando se acumula más tensión y se desencadena una menor autoestima sobre todo en las chicas (Smith, Cénat, Lapierre, Dion, Hébert y Côté, 2018). Aunque las diferencias entre los adolescentes hombres y mujeres al ejercer la violencia sean pequeñas, hay estudios que sostienen que las chicas presentan actitudes celosas y ejercen más la violencia verbal, entre el 18.5 y el $90.3 \%$ frente a los chicos que las muestran entre el 8.1 y el 83.3 \% (Sebastián et al., 2014). Por otro lado, otros estudios sostienen que los chicos perpetran más la violencia física y coerción sexual (Cañete y Novas, 2012; Saldivia, y Vizcarra, 2012; Viejo, 2012).

Mientras la violencia física en las citas se pone de manifiesto a través de comportamientos violentos (por ejemplo, puñetazos), intimidación física o el uso de objetos con la finalidad de hacer daño (lanzar objetos a la pareja) (Yan et al., 2009), la violencia psicológica es difícil de percibir por su apariencia amorosa: conductas restrictivas y controladoras que interfieren en la capacidad de decisión y autonomía de los adolescentes (Yugueros, 2012), por lo que se tiende a manipular, ganar poder o control sobre la pareja (Martine, Martin y Francine, 2017; Mueller, Jouriles, McDonald y Rosenfield, 2013). Estas conductas pueden conducir a los adolescentes a desarrollar ansiedad, depresión, enfermedad mental, trastornos por consumo de sustancias y suicidio (Beatriz, Lincoln, Alder, Daley, Simmons, Ibeh, Figueroa y Molnar, 
2018; Parker, Johnson, Debnam, Milam y Bradshaw, 2017; Temple, Choi, Reuter, Wolfe, Taylor, Madigan y Scott, 2018), lo cual fue evidenciado en una encuesta realizada en la ciudad de Nueva York en 2005, en la que se observó que el $7.2 \%$ de chicos y el $11.7 \%$ de chicas habían cometido uno o más intentos de suicidio a causa de la violencia psicológica perpetrada por la pareja (Holmes y Sher, 2013). La violencia psicológica ejercida entre las parejas jóvenes en España oscila entre el 4 y el $97 \%$, según reveló una revisión sistemática de 1221 referencias (Garay, González, Carrasco y Amor, 2017). A diferencia de otros tipos de violencia, un estudio mostró niveles más altos de violencia psicológica perpetrada por los estudiantes en los dos últimos años de Instituto (Fernández-González, O’Leary y Muñoz-Rivas, 2013).

Las relaciones y la comunicación de la pareja con los otros a través del móvil y redes sociales pueden ser objeto de violencia durante el noviazgo. Esta modalidad de violencia sobre la pareja se ejerce en público o en privado por medio de las redes virtuales; comprende acciones de abuso, control, vigilancia, manifestaciones de celos, enviar materiales digitales privados sin permiso, propagación de rumores insultantes, degradantes, humillantes o amenazantes hacia la pareja, exclusión social de grupos reales y virtuales, revisión de correos electrónicos, WhatsApp, petición de contraseñas y acoso sexual (Burke, Wallen, Vail-Smith y Knox, 2011; Draucker y Martsolf, 2010; Martinez-Pecino y Durán, 2016; Picard, 2007).

Las conductas violentas de los adolescentes y jóvenes también pueden ser aprendidas por exposición previa a la violencia o vivir situaciones familiares en las que esta se ejerce, lo que hace que los menores repitan las conductas violentas vividas (Cohen, Shorey, Menon y Temple, 2018; Lee, Reese-Weber y Kahn, 2014; Livingston, Eiden, Lessard, Casey, Henie y Leonard, 2018; Reidy, Holland, Cortina, Ball y Rosenbluth, 2017).

Para combatir la violencia en el noviazgo los expertos sugieren una mayor educación que armonice lo individual y social informando sobre la solución de conflictos entre los jóvenes (Rodríguez et al., 2013; Shorey, Wymbs, Torres, Cohen, Fite y Temple, 2017). De hecho, muchos programas van en el sentido de la prevención de la violencia en las parejas adolescentes, empoderando a jóvenes que actúan como líderes en los grupos y transmiten valores positivos a sus compañeros (Abebe, Jones, Ciaravino, Ripper, Paglisotti, Morrow y Miller, 2017; Liu, Mumford y Taylor, 2018; Rodríguez, Ramos, Contreras y Cáceres, 2013).

La satisfacción con la vida es uno de los tres elementos que componen el constructo bienestar subjetivo (Ponce-Díaz, Aiquita y Arboccó, 2019; Rodriguez-Fernández, Ramos-Díaz, Ros, Fernández-Zabala y Revuelta, 2016) y se define como la evaluación cognitiva y global de la calidad de la vida en general. Estudios han evidenciado que, como consecuencia de la violencia en las relaciones de pareja, se produce una disminución del nivel de satisfacción con la vida en la persona maltratada (García, Wlodarezyk, Reyes, San Cristobal y Soler, 2014).

\section{Conductas sexistas y violencia en la pareja}

A través de los procesos de socialización, los seres humanos van construyendo su propia identidad e incorporando los comportamientos, las características y los roles que la sociedad atribuye a los individuos en función de su sexo (Rouyer, Mieyaa y le Blanc, 2014); se trata de conductas culturalmente aprendidas. La violencia hacia la mujer es un problema complejo, social e histórico. Se ha ejercido violencia hacia la mujer solo por el hecho de serlo, y una explicación que se da para ello es el modelo de sociedad jerarquizada con el poder en manos de los hombres, que nunca han favorecido cambios ante estas creencias sexistas (Abebe, Jones, Culyba, Feliz, Anderson, Torres y Miller, 2018; Erdem 
y Sahin, 2017; Montolío, Ros y Portela, 2016).

Los adolescentes que han sido educados en el marco de conductas sexistas o comportamientos propios de sociedades patriarcales son más tolerantes con el abuso físico o psicológico en sus relaciones de pareja y es menos probable que identifiquen los comportamientos agresivos o violentos con formas de violencia (Shaffer, Corona, Sullivan, Fuentes y McDonald, 2018).

Investigaciones recientes han venido a constatar la existencia de estereotipos de género entre los estudiantes de educación secundaria. Un estudio realizado por Miller, Eagly y Linn (2015) a estudiantes universitarios de 66 países reveló que los hombres consideraban que estaban más capacitados que las mujeres para los trabajos científico-tecnológicos. Por otro lado, Rodríguez, Lameiras, Carrera y Faílde (2010) evidenciaron la existencia de un elevado nivel de sexismo interiorizado hacia las mujeres estudiantes de educación secundaria. Estas creencias resultan inquietantes en el campo de la sexualidad y las relaciones amorosas, por su implicación en la justificación de actitudes y conductas violentas hacia las parejas.

\section{Religión y violencia en las relaciones de pareja}

Las creencias religiosas forman parte de la formación de la persona y contribuyen a la formación de su identidad personal; son también producto de las características socioculturales y resaltan los roles de género y de pareja (Armet, 2009; Vermeer, 2014).

Atendiendo a la teoria del control social (Hirschi, 1969), se ha constatado que las familias que educan a sus hijos en creencias religiosas y en la adquisición de compromisos logran que estos jóvenes incrementen su vínculo social al aumentar la aceptación de las normas socialmente establecidas, por lo que la religión se convertiría en un protector contra las conductas violentas y socialmente condenadas (Mitchell, 2019).
Según el Observatorio de Pluralismo Religioso (2013), en España, más del $45 \%$ de la población afirma que la religión es muy sustancial en sus vidas. El $67 \%$ son creyentes y el $57.5 \%$ educarían a sus hijos en la doctrina de una religión. Un estudio con 6621 jóvenes españoles de entre 15 y 26 años resaltó que los estudiantes que practicaban una religión declaraban menos violencia con su pareja, desde el 3.76 y $74 \%$, frente a los estudiantes que no eran creyentes que manifestaban una prevalencia entre el 4.11 y el $83 \%$ (Fernández, Rodríguez, Molleda y Rodríguez, 2015). Estos mismos datos son usados por un estudio que afirma que jóvenes menos creyentes sufren las distintas formas de violencia de pareja en proporciones superiores a los jóvenes que sí practican alguna religión (Sanabria, Jiménez, Parra, Blandón y Tordecilla, 2016). Se defiende desde esta posición que la religión puede actuar como un sistema de significado o como una estrategia de afrontamiento de problemas que surjan en las parejas jóvenes, lo cual explica que la espiritualidad ayuda a comprender a la pareja (Wendt y Zannettino, 2015). Aunque estos datos revelan que las creencias religiosas podían actuar como un recurso protector de la violencia en la pareja, hay que seguir profundizando en las investigaciones.

Con esta investigación se pretende conocer la incidencia de la violencia fisica, psicológica y comunicativa en los adolescentes, detectar las conductas sexistas en los jóvenes de ambos sexos y determinar cómo perciben los adolescentes, hombres y mujeres, la violencia y la relación de esta con la satisfacción con la vida. Por último, se busca conocer si la religión puede actuar como protectora de la violencia y su incidencia en la satisfacción con la vida de los adolescentes.

\section{Método}

\section{Participantes}

Formaron parte del estudio 1036 adolescentes de entre 14 y 19 años, de los cuales el 
$48.1 \%$ eran hombres y el $51.9 \%$ mujeres. El $64 \%$ eran cristianos, el $7.6 \%$, islámicos, el 0.2 $\%$, judíos, el $0.5 \%$, budistas, el $1.1 \%$, agnósticos, el $14.7 \%$, ateos y el $11.9 \%$ no contestaron la pregunta. Al momento del estudio, el $36.6 \%$ tenía pareja. Los adolescentes participantes pertenecían a siete centros de Educación Secundaria Obligatoria y Bachillerato del sureste español y, como requisito, debían tener una relación de pareja o haberla tenido en los últimos seis meses.

\section{Procedimiento}

Se realizó un estudio descriptivo transversal y predictivo, con un muestreo por conglomerados, con selección aleatoria de los centros facilitados por la administración, con el requisito de que hubiera en ellos presencia de alumnado inmigrado. Una vez elegidos los centros y antes de proceder a la recogida de datos, se solicitó permiso a los equipos directivos y a los padres/madres. Antes de aplicar las pruebas, se obtuvieron el permiso y el consentimiento informado ya firmado. Cuando se procedió a la aplicación de la prueba en las aulas, se ofrecieron datos de procedimiento de la investigación y se reservó un tiempo para aclarar dudas; a continuación, se aplicaron las escalas en formato papel en las aulas y en presencia del profesor tutor al alumnado de 3. ${ }^{\mathrm{a}}$ de la Educación Secundaria Obligatoria y 2. ${ }^{\mathrm{a}}$ de bachillerato. A fin de garantizar el anonimato de la prueba, se requirió a los participantes que no escribieran datos que pudieran servir para identificarlos y se informó que los resultados serían obtenidos sobre el conjunto, nunca sujeto a sujeto.

\section{Instrumentos}

Además de recabar datos personales (edad, género, religión y situación sentimental), se aplicaron las siguientes pruebas:

Escala de Satisfacción con la Vida (SWLS) de Diener, Emmons, Larsen y Griffin (1985). Es una escala tipo Likert en la que el rango de respuesta va de 1 (totalmente de acuerdo) a 5 (totalmente en desacuerdo). Está formada por cinco ítems y evalúa los aspectos cognitivos del bienestar; de tal forma que a menor puntuación más satisfacción con la vida. Esta escala arrojó un alfa de Cronbach de .733 .

Las Escalas de Violencia Psicológica, Física, Comunicación y Relación con los demás y la Escala de Conductas Sexistas, han sido elaboradas y validadas por Aguilera y Soriano (2017). Para ello, las autoras han formulado ítems y otros han sido adaptados de la Escala de Violencia en la Pareja y Escala sobre justificación, violencia y sexismo del Ministerio de Sanidad, Servicios Sociales e Igualdad del Gobierno de España en 2014.

La Escala de Violencia Psicológica es tipo Likert con un rango de respuesta desde 1 (nunca) hasta 4 (siempre) y consta de ocho ítems. Esta escala evalúa las conductas que producen desvalorización y sufrimiento emocional. Una de las preguntas es, por ejemplo: "¿Te ha insultado o ridiculizado?". La escala arrojó un alfa de Cronbach de .777 .

La Escala de Violencia Física es tipo Likert y puntúa de 1 (nunca) a 4 (siempre). Está formada por seis ítems y evalúa la violencia física e impresión de autoridad. Por ejemplo, “¿Cuándo se enfada, te pellizca?”. Esta escala obtuvo una fiabilidad de.819.

La Escala sobre Violencia en la Comunicación y Relación con los demás también es tipo Likert, con rango de respuesta de 1 (nunca) a 4 (siempre) y está formada por cinco ítems. Entre las preguntas: "¿Ha intentado mantenerte aislado de amistades?”, “¿Dice que es necesario compartir contraseñas de redes sociales como Facebook, mail, Twitter?". La fiabilidad de la escalafue de.757.

El cuestionario sobre Conductas Sexistas presenta respuestas dicotómicas, está 
compuesto por nueve ítems y evalúa los mitos, las falsas creencias y las conductas sexistas. Las siguientes son ejemplos de preguntas: "¿Cuando una mujer es agredida por su pareja, algo habrá hecho para provocarlo?”, “¿Está justificado que un hombre agreda a su mujer o a su novia cuando ella decide dejarle?".

\section{Criterios éticos}

La investigación fue aprobada por el Comité de Bioética de la Universidad de Almería. Los adolescentes invitados a participar eligieron con toda libertad realizar el estudio y se les informó sobre la naturaleza, duración y propósito del mismo. El procedimiento siguió las normas como viene recogido en la Convención de Helsinki (2013) para investigaciones científicas en las que se incluyan participantes humanos.

\section{Análisis de datos}

Los datos recogidos fueron analizados mediante el paquete estadístico SPSS (Statistical Package for the Social Sciences) versión 23 para Windows. Se realizó la prueba de normalidad de Shapiro-Wilk porque Pedrosa, Juarros, Robles, Basteiro y García-Cueto (2015) consideran que es la más precisa para muestras superiores a 500 sujetos y se comprobó que la distribución de los datos no se ajustaba a una normal. Se calculó la correlación entre las distintas escalas de violencia y la satisfacción con la vida; para este cálculo en concreto se recodificaron los ítems que componen la escala de satisfacción con la vida indicando que 1 es totalmente en desacuerdo y 5 , totalmente de acuerdo, para que fuese en el mismo sentido que las tres variables de violencia. Además, se calculó la $U$ de Mann Whitney para muestras independientes, prueba de Kruskal-Wallis, tamaño del efecto a través de la $r$ de Rosenthal y regresiones logísticas con las conductas sexistas. Para las regresiones logísticas, las variables dependientes fueron cada una de las conductas sexistas, considerando independientes el género y la práctica religiosa. En las variables dependientes a la categoría de referencia se le asigna 0 y 1 a la que se considera de riesgo. En la variable independiente se consideró categoría de referencia ser hombre (0) para el sexo y ser practicante para la religión.

\section{Resultados}

\section{Estadística descriptiva y correlaciones de las escalas}

Se calcularon las medias y desviaciones estándar de las escalas de violencia en la comunicación, violencia psicológica, violencia física y satisfacción con la vida, y se obtuvieron las correlaciones entre variables (Tabla 1).

Tabla 1

Medidas descriptivas de las escalas estudiadas y correlación entre ellas.

\begin{tabular}{c|cccc} 
& $\begin{array}{c}\text { Violencia en la } \\
\text { comunicación }\end{array}$ & $\begin{array}{c}\text { Violencia } \\
\text { psicológica }\end{array}$ & $\begin{array}{c}\text { Violencia } \\
\text { física }\end{array}$ & $\begin{array}{c}\text { Satisfacción } \\
\text { con la vida }\end{array}$ \\
\hline$M$ & 1.40 & 1.41 & 1.27 & 2.60 \\
$D S$ & .514 & .429 & .451 & .884 \\
$\begin{array}{c}\text { Violencia en la } \\
\text { comunicación }\end{array}$ & 1 & & & \\
$\begin{array}{c}\text { Violencia psico- } \\
\text { lógica }\end{array}$ & $.461^{* *}$ & 1 & & \\
& & & &
\end{tabular}




\begin{tabular}{c|cccc} 
& $\begin{array}{c}\text { Violencia en la } \\
\text { comunicación }\end{array}$ & $\begin{array}{c}\text { Violencia } \\
\text { psicológica }\end{array}$ & $\begin{array}{c}\text { Violencia } \\
\text { física }\end{array}$ & $\begin{array}{c}\text { Satisfacción } \\
\text { con la vida }\end{array}$ \\
\hline $\begin{array}{c}\text { Violencia física } \\
\text { Satisfacción con }\end{array}$ & $.409^{* *}$ & $.520^{* *}$ & 1 & \\
la vida & $-.125^{* *}$ & $-.210^{* *}$ & $-.124 * *$ & 1
\end{tabular}

$* * p=.000$

La puntuación media de la escala de satisfacción con la vida es de 2.60; al ser el rango de respuesta en la escala de 1 a 5 , se puede interpretar como un grado intermedio en la satisfacción de los adolescentes con la vida y con sus expectativas. Por otro lado, las puntuaciones en las escalas de violencia psicológica, en la comunicación y física, que tienen un rango de respuesta de 1 a 4, indican que no existen niveles elevados de violencia entre los adolescentes.

La correlación más alta (.520) se obtiene entre la escala de violencia psicológica y la física. Se observa correlación negativa entre las escalas de violencia física, psicológica y de comunicación con respecto a la satisfacción personal. Es decir, a más violencia sufrida, menor satisfacción con la vida.

\section{Conductas sexistas}

La Tabla 2 muestra las regresiones logísticas de las conductas sexistas con los predictores género y práctica religiosa.

Tabla 2

Porcentajes y regresión logística de las conductas sexistas según sexo y práctica religiosa.

\begin{tabular}{|c|c|c|c|c|c|c|c|c|}
\hline & \multicolumn{4}{|c|}{ Conducta } & \multicolumn{4}{|c|}{ Regresiones logísticas } \\
\hline & \multicolumn{2}{|c|}{ Sexo } & \multicolumn{2}{|c|}{ Practicante } & \multicolumn{2}{|c|}{ Sexo } & \multicolumn{2}{|c|}{ Practicante } \\
\hline & $\begin{array}{c}\text { H } \\
\text { n (\%) }\end{array}$ & $\begin{array}{c}\text { M } \\
\text { n (\%) }\end{array}$ & $\begin{array}{c}\text { Si } \\
\text { n (\%) }\end{array}$ & $\begin{array}{c}\text { No } \\
\text { n (\%) }\end{array}$ & $\boldsymbol{\beta}$ & $\begin{array}{c}\text { OR } \\
\text { IC } 95 \%\end{array}$ & $\beta$ & $\begin{array}{c}\text { OR } \\
\text { IC } 95 \%\end{array}$ \\
\hline $\begin{array}{l}\text { Está bien que los chicos } \\
\text { salgan con muchas chicas } \\
\text { pero no al revés. }\end{array}$ & $\begin{array}{c}110 \\
(22.1)\end{array}$ & $\begin{array}{c}23 \\
(4.3)\end{array}$ & $260(85)$ & $\begin{array}{c}643 \\
(88.1)\end{array}$ & 1.398 & $\begin{array}{c}\mathbf{4 . 0 4 7 * *} \\
1.969-8.319\end{array}$ & -.059 & $\begin{array}{c}.060 \\
.727-1.223\end{array}$ \\
\hline $\begin{array}{l}\text { A veces hay que amenazar } \\
\text { a los demás para que sepan } \\
\text { quién es el que manda. }\end{array}$ & $\begin{array}{l}154 \\
(31)\end{array}$ & $\begin{array}{c}92 \\
(17.1)\end{array}$ & $\begin{array}{c}230 \\
(75.2)\end{array}$ & $\begin{array}{c}559 \\
(76.6)\end{array}$ & .776 & $\begin{array}{c}\mathbf{2 . 1 7 3}^{*} \\
1.274-3.706\end{array}$ & -.109 & $\begin{array}{c}1.116 \\
.716-1.122\end{array}$ \\
\hline $\begin{array}{c}\text { Por el bien de sus hijos, } \\
\text { aunque la mujer u hombre } \\
\text { tenga que soportar la } \\
\text { violencia de su pareja, } \\
\text { conviene que no le denuncie. }\end{array}$ & $\begin{array}{c}100 \\
(20.1)\end{array}$ & $\begin{array}{c}122 \\
(22.7)\end{array}$ & $\begin{array}{c}243 \\
(79.4)\end{array}$ & $\begin{array}{c}571 \\
(78.2)\end{array}$ & -.223 & $\begin{array}{c}1.25 \\
.458-1.397\end{array}$ & .062 & $\begin{array}{c}1.064 \\
.889-1.274\end{array}$ \\
\hline $\begin{array}{l}\text { Si una mujer u hombre es } \\
\text { maltratado/a por su compa- } \\
\text { ñero/a y no le abandona será } \\
\text { porque no le disgusta del } \\
\text { todo la situación. }\end{array}$ & $\begin{array}{l}164 \\
(33)\end{array}$ & $\begin{array}{c}123 \\
(22.9)\end{array}$ & $\begin{array}{c}223 \\
(79.2)\end{array}$ & $\begin{array}{c}526 \\
(72.1)\end{array}$ & .429 & $\begin{array}{c}1.536 \\
.924-2.553\end{array}$ & .065 & $\begin{array}{c}1.067 \\
.852-1.338\end{array}$ \\
\hline
\end{tabular}




\begin{tabular}{|c|c|c|c|c|c|c|c|c|}
\hline & \multicolumn{4}{|c|}{ Conducta } & \multicolumn{4}{|c|}{ Regresiones logísticas } \\
\hline & \multicolumn{2}{|c|}{ Sexo } & \multicolumn{2}{|c|}{ Practicante } & \multicolumn{2}{|c|}{ Sexo } & \multicolumn{2}{|c|}{ Practicante } \\
\hline & $\begin{array}{c}\text { H } \\
\text { n (\%) }\end{array}$ & $\begin{array}{c}\text { M } \\
\text { n (\%) }\end{array}$ & $\begin{array}{c}\text { Si } \\
\text { n (\%) }\end{array}$ & $\begin{array}{c}\text { No } \\
\text { n (\%) }\end{array}$ & $\boldsymbol{\beta}$ & $\begin{array}{c}\text { OR } \\
\text { IC } 95 \%\end{array}$ & $\boldsymbol{\beta}$ & $\begin{array}{c}\text { OR } \\
\text { IC } 95 \%\end{array}$ \\
\hline $\begin{array}{l}\text { Cuando una mujer es agre- } \\
\text { dida por su pareja, algo habrá } \\
\text { hecho ella para provocarlo. }\end{array}$ & $\begin{array}{c}136 \\
(27.4)\end{array}$ & $\begin{array}{c}64 \\
(11.9)\end{array}$ & $\begin{array}{c}244 \\
(79.7)\end{array}$ & $\begin{array}{c}592 \\
(81.1)\end{array}$ & 1.264 & $\begin{array}{c}\mathbf{3 . 5 3 8}^{* *} \\
1.916-6.532\end{array}$ & -.147 & $\begin{array}{c}1.158 \\
.666-1.118\end{array}$ \\
\hline $\begin{array}{c}\text { Para tener una buena relación } \\
\text { de pareja es deseable que } \\
\text { la mujer evite llevarle la } \\
\text { contraria al hombre. }\end{array}$ & $\begin{array}{c}74 \\
(14.9)\end{array}$ & $43(8)$ & $\begin{array}{c}269 \\
(87.9)\end{array}$ & $\begin{array}{l}650 \\
(89)\end{array}$ & .488 & $\begin{array}{c}1.628 \\
.810-3.275\end{array}$ & -.019 & $\begin{array}{c}1.019 \\
.727-1.325\end{array}$ \\
\hline $\begin{array}{l}\text { La violencia que se produce } \\
\text { dentro de la casa es un asunto } \\
\text { de familia y no debe salir de } \\
\text { ahí. }\end{array}$ & $\begin{array}{c}141 \\
(28.4)\end{array}$ & $\begin{array}{c}132 \\
(24.6)\end{array}$ & $\begin{array}{c}222 \\
(72.5)\end{array}$ & $\begin{array}{c}541 \\
(74.1)\end{array}$ & .527 & $\begin{array}{c}1.694 * \\
1.019-2.816\end{array}$ & -.066 & $\begin{array}{c}1.068 \\
.763-1.148\end{array}$ \\
\hline $\begin{array}{c}\text { Está justificado que un } \\
\text { hombre agreda a su mujer o } \\
\text { a su novia cuando ella decide } \\
\text { dejarle. }\end{array}$ & $\begin{array}{c}62 \\
(12.5)\end{array}$ & $\begin{array}{c}60 \\
(11.2)\end{array}$ & $\begin{array}{c}272 \\
(88.9)\end{array}$ & $\begin{array}{c}642 \\
(87.9)\end{array}$ & .318 & $\begin{array}{c}1.374 \\
.670-2.816\end{array}$ & .198 & $\begin{array}{c}1.219 \\
.863-1.723\end{array}$ \\
\hline $\begin{array}{l}\text { Alguna que otra vez se puede } \\
\text { aceptar que un chico o chica } \\
\text { insulte y/o dé una torta a su } \\
\text { pareja si se lo ha merecido. }\end{array}$ & $\begin{array}{c}161 \\
(32.4)\end{array}$ & $\begin{array}{c}153 \\
(28.5)\end{array}$ & $\begin{array}{c}212 \\
(69.3)\end{array}$ & $\begin{array}{c}509 \\
(69.7)\end{array}$ & -.151 & $\begin{array}{c}1.162 \\
.528-1.402\end{array}$ & .048 & $\begin{array}{c}1,049 \\
.852-1.291\end{array}$ \\
\hline
\end{tabular}

$* \mathrm{p}<.05 ; * * \mathrm{p}<.000$.

Las pruebas de Hosmer y Lemeshow no son estadísticamente significativas en los modelos de regresiones logísticas realizados. En el primero de estos modelos ("Está bien que los chicos salgan con muchas chicas, pero no al revés"; wald $=14.461, \mathrm{p}=.000$, $\mathrm{OR}=4.047$ ), se observa que es 4.047 veces más probable que los chicos estén de acuerdo con esta actitud que las chicas. Asimismo, es estadísticamente significativo el ítem "A veces hay que amenazar a los demás para que sepan quién es el que manda" (wald $=8.107$, $\mathrm{p}=.004, \mathrm{OR}=2.173$ ), lo que indica que es 2.173 veces más probable que los chicos estén de acuerdo con esa conducta que las chicas. También es estadísticamente significativo el ítem "Cuando una mujer es agredida por su

pareja algo habrá hecho ella para provocarlo" (wald $=16.315, \mathrm{p}=.000, \mathrm{OR}=.538$ ), por lo que es 3.538 veces más probable que los chicos respalden esta actitud que las chicas. A su vez, es estadísticamente significativo el enunciado "La violencia que se produce dentro de la casa es un asunto de familia y no debe salir de ahí" (wald $=4.13, \mathrm{p}=.042$, $\mathrm{OR}=1.694)$, lo que indica también que es 1.694 veces más probable que los chicos adopten este comportamiento que las chicas. No obstante, no se encontraron diferencias al comparar las distintas conductas sexistas de la escala cuando utilizamos como predictor ser practicante de alguna religión. 


\section{Violencia y género}

Para analizar las posibles diferencias en los tipos de violencia entre los dos sexos, se calcularon rangos, se realizó la prueba de Mann Whitney y se calculó el tamaño del efecto a través de la $\mathrm{r}$ de Rosenthal (Tabla 3).

Tabla 3

Rangos, prueba de Mann Whitney y tamaño del efecto en los distintos tipos de violencia.

\begin{tabular}{c|c|c|c|c|c|c} 
& $\begin{array}{c}\text { Hombres } \\
\mathbf{R}\end{array}$ & $\begin{array}{c}\text { Mujeres } \\
\mathbf{R}\end{array}$ & $\mathbf{U}$ & $\mathbf{Z}$ & $\mathbf{p}$ & $\mathbf{r}$ \\
\hline V. comunicación & 536.22 & 500.17 & 12478 & -1.95 & $\mathbf{. 0 5}$ & -.06 \\
V. psicológica & 553.86 & 483.85 & 115970.5 & -3.78 & $\mathbf{. 0 0 0}$ & -.11 \\
V. física & 578.84 & 460.73 & 103431.5 & -6.76 & $\mathbf{. 0 0 0}$ & -.21 \\
Satisfacción & 491.72 & 541.36 & 121388 & -2.61 & .009 & -.08
\end{tabular}

Los resultados son significativos en los tres tipos de violencia: comunicación, psicológica y física, y los hombres obtuvieron rangos más altos que las mujeres, lo que sugiere que, aunque los niveles generales de violencia sufrida no son altos, sí se observa que los hombres perciben sufrir más violencia que las mujeres (Tabla 3). La satisfacción con la vida también arroja resultados significativos. En este caso los hombres perciben mayor satisfacción con su vida.

\section{Violencia en la pareja y práctica religiosa}

Para los estudiantes con creencias y prácticas religiosas, se evidenciaron resultados estadísticamente significativos solo en la satisfacción con la vida, mostrando que los estudiantes que practican una religión tienen más satisfacción con la vida, aunque el tamaño del efecto es muy pequeño (Tabla 4).

\section{Tabla 4}

Rangos, prueba de Mann Whitney y tamaño del efecto en los distintos tipos de violencia considerando la religión variable independiente.

\begin{tabular}{c|c|c|c|c|c|c} 
& \multicolumn{2}{|c|}{$\boldsymbol{R}$ Practicante } & $\mathbf{U}$ & $\mathbf{Z}$ & $\mathbf{p}$ & $\mathbf{r}$ \\
& Sí & No & & & & \\
\hline V. Comunicación & 530.32 & 512.14 & 108008 & -.86 & .389 & -.02 \\
V. Psicológica & 519.19 & 516.79 & 111416.5 & -.06 & .950 & -.00 \\
V. Física & 526.66 & 513.67 & 109005.5 & -.65 & .515 & -.02 \\
Satisfacción vida & 467.24 & 538.53 & 96666 & -3.42 & $\mathbf{. 0 0 1}$ & -.10
\end{tabular}

\section{Discusión}

Este estudio analiza la violencia sufrida en las parejas adolescentes que viven en el sureste español, sus actitudes y conductas sexistas, la asociación entre violencia física, psicológica, en la comunicación y la satisfacción de los jóvenes con la vida, y el papel de la religión como posible protector contra la violencia.

La satisfacción con la vida como un elemento 
del constructo de la felicidad subjetiva correlaciona negativamente con la violencia física, con la violencia que se produce en la comunicación, pero alcanza correlación mayor con la violencia psicológica. La percepción y el sentimiento de ser maltratado hace que disminuya la satisfacción con la vida, resultados que son coherentes con el estudio realizado por García, Wlodarezyk, Reyes, San Cristobal y Soler (2014).

Las conductas sexistas se muestran en los adolescentes de este estudio, lo cual coincide con los resultados hallados por Rodríguez, Lameiras, Carrera y Faílde (2010) y Miller, Eagly y Linn (2015). Los adolescentes hombres ven como normal que la sociedad no apruebe que una chica pueda salir con muchos hombres, pero que sí sea bien visto que un hombre pueda salir con muchas mujeres. También perciben como normal el control y la amenaza sobre la "otra" como acto de autoridad, incluso indicando que cuando una mujer es agredida por su pareja es que ella lo ha provocado; a la vez los hombres adolescentes entienden que la violencia dentro del hogar no debe conocerse afuera. Diversos autores (Abebe, Jones, Culyba, Feliz, Anderson, Torres y Miller, 2018; Cañete y Novas, 2012; ErdemySahin, 2017; Montolío, Ros y Portela, 2016; Santos, 2017) explican que el sexismo se basa en el mantenimiento del poder y de una identidad distintiva por parte de los hombres respecto a las mujeres, junto a deseos ambivalentes de intimidad y dominación sexual, manifestando un modelo de sociedad jerarquizada, lo que concuerda con nuestros resultados que reflejaron falsas creencias y conductas sexistas con más fuerza en los varones. Además, se coincide con Shaffer, Corona, Sullivan, Fuentes y McDonald (2018) y Gómez, Barrientos, Guzmán, Cárdenas y Bahamondes (2017) en el hecho de que los adolescentes que han sido educados asumiendo conductas sexistas son incapaces de identificar los comportamientos agresivos como forma de violencia. Los resultados hallados, además, hacen reflexionar acerca de cómo, viviendo en una sociedad científica y tecnológicamente avanzada, los niveles de sexismo entre los adolescentes sean altos.

En la violencia en la pareja se detecta un integrante que actúa perpetrando y otro que se comporta como víctima. En diversos estudios se ha presentado al hombre como agresor y a la mujer como víctima de esta violencia (Abebe et al., 2018; Cañete y Novas, 2012; Erdem y Sahin, 2017; Montolío et al., 2016), y en otros, a la mujer en el papel de perpetradora con niveles iguales o superiores al hombre (Reidy, Kearns, Houry, Valle, Holland y Marshall, 2016; Sebastián et al., 2014). Estos últimos estudios concuerdan con los resultados obtenidos en esta investigación, en los que el adolescente hombre percibe sufrir más violencia que las adolescentes mujeres en los tres tipos de violencia estudiados: física, psicológica y en la comunicación/relación. Sin embargo, los resultados no son consistentes con la investigación de Chan (2012), quien encontró que las mujeres recibían de media más violencia física, emocional y verbal en comparación con los hombres y perpetraban más la violencia psicológica grave. Según defiende el estudio de Alegría del Ángel y Rodríguez (2015), estos cambios pueden deberse a que la sociedad está mostrando cambios socioculturales y rompiendo con la idea clásica de género, sobre todo en lo que respecta al rol de sumisión de la mujer que tradicionalmente se le había impuesto, aumentando la probabilidad de la violencia en el seno de la pareja. Este cambio de posición de las mujeres es lo que está llevando a un modelo bidireccional de la violencia en las relaciones de pareja en los adolescentes. No obstante, aunque los hombres adolescentes perciben más violencia por parte de sus parejas, son ellos los que muestran más conductas sexistas que las mujeres, lo que invita a seguir investigando y analizar la asunción, también, de las conductas sexistas por parte de las mujeres que podrían actuar normalizando la violencia.

Por otro lado, según Smith et al. (2018), las mujeres sufrían más cambios psicológicos al inicio de la relación de pareja, angustia y de 
media menor percepción de condiciones de vida próximas a su ideal. Estos datos trataban de explicar los hallazgos de una investigación que reflejó mayores porcentajes de suicidios en las mujeres con respecto a los hombres (Holmes y Sher, 2013). Los resultados de este estudio han revelado que los hombres se sienten mas satisfechos con la vida que las mujeres, aunque los hombres perciben recibir más violencia que las mujeres. Sin embargo, son diferencias muy leves entre los distintos sexos.

Por otra parte, hay estudios que respaldan que los jóvenes menos religiosos ejercían de media mayores niveles de violencia de todo tipo, y defendían que la religión puede actuar como una estrategia de afrontamiento de problemas en las parejas jóvenes porque ayuda a entender la espiritualidad de la pareja (Wendt y Zannettino, 2015). Sin embargo, en contraste con estas investigaciones, los resultados de esta investigación no muestran diferencias en la violencia física, psicológica y en la comunicación sufridas entre los distintos grupos de estudiantes atendiendo a la variable religión. Pero sí se ha mostrado que los jóvenes practicantes (independientemente de la religión) se sienten más satisfechos con la vida que los no practicantes. Del mismo modo, otros estudios (Armet, 2009; Vermeer, 2014) tratan de explicar las características socioculturales, los roles de género y de pareja con las creencias religiosas, resaltando el estado de bienestar de los jóvenes practicantes con respecto de los no practicantes.

Como limitación del presente estudio se señala que solo se pregunta a los adolescentes si son practicantes y la religión que practican, pero no se profundiza en preguntas que informen sobre cómo influye la religión en sus vidas y cómo la entienden. En futuras investigaciones es necesario profundizar más ampliamente en la religión y en los valores morales, para complementar estudios cuantitativos sobre el tema con estudios cualitativos.

En conclusión, el género, la satisfacción con la vida, el sexismo y ser practicante religioso son variables trascendentes a la hora de estu- diar la reciprocidad de la violencia en parejas jóvenes y adolescentes. Igualmente se perciben falsas creencias y sexismo, sobre todo en los adolescentes hombres. Por lo tanto, es necesario crear programas de educación sexual para los adolescentes que contemplen la afectividad en las relaciones, detecten el sexismo y la violencia, y se trabaje en este sentido para que sean capaces de reconocer la violencia y no asumir papeles pasivos de víctimas. Es importante investigar sobre la espiritualidad y/o los valores, y profundizar en su posible carácter protector contra la violencia.

\section{Referencias}

Aguilera, O. y Soriano, E. (2017). Violencia en las relaciones de pareja en la adolescencia. Relación con la satisfacción con la vida [Trabajo de fin de Master]. Universidad de Almería, España. Alegría del Ángel, M. y Rodríguez Barraza, A. (2015). Violencia en el noviazgo: Perpetración, victimización y violencia mutua. Una revisión. Actualidades en Psicología, 29(118), 57-72. https://doi.org/10.15517/ap.v29i118.16008

Abebe, K. Z., Jones, K. A, Ciaravino, S., Ripper, L., Paglisotti, T., Morrow, S. E.y Miller, E. (2017). A cluster-randomized trial of a middle school gender violence prevention program: Design, rationale, and sample characteristics. Contemporary Clinical Trials, 62, 11-20. https://doi. org/10.1016/j.cct.2017.08.007

Abebe, K. Z., Jones, K. A., Culyba, A. J., Feliz, N. B., Anderson, H., Torres, I. y Miller, E. (2018). Engendering healthy masculinities to prevent sexual violence: Rationale for and design of the Manhood 2.0 trial. Contemporary Clinical Trials, 71, 18-32. https://doi.org/10.1016/j. cct.2018.05.017

Armet, S. (2009). Religious socialization and identity formation of adolescents in high tension religions. Review of Religious Research, 50, 277-29.

Beatriz, E. D., Lincoln, A. K., Alder, J., Daley, N., Simmons, F., Ibeh, K., Figueroa, C. y Molnar, B. E. (2018). Evaluation of a Teen Dating Violence Prevention Intervention among Urban 
Middle-School Youth Using Youth Participatory Action Research: Lessons Learned from Start Strong Boston. Journal of Family Violence, 33, 563-578. https://doi.org/10.1007/s10896-0189981-4

Burke, S. C., Wallen, M., Vail-Smith, K. y Knox, D. (2011). Using technology to control intimate partners: An exploratory study of college undergraduates. Computers in Human Behavior, 27(3), 1162-1167. https://doi.org/10.1016/j. chb.2010.12.010

Cañete, E. P. y Novas, F. P. (2012). Resolución de conflictos de pareja en adolescentes, sexismo y dependencia emocional. Quaderns de Psicologia, 14(1), 45-60. https://raco. cat/index.php/QuadernsPsicologia/article/ view/10.5565-rev-psicologia.1041/341248

Casabona, N. M. y Tellado, I. (2012). Violencia de Género y Resolución Comunitaria de Conflictos en los Centros Educativos. Multidisciplinary Journal of Gender Studies, 1(3), 300-319. https://doi.org/10.4471/generos.2012.14

Centers for Disease Control and Prevention. (2012). Understanding teen dating violence fact sheet. http://www.cdc.gov/ViolencePrevention/pdf/ TeenDating-violence2012-a.pdf

Chan K. L. (2012). The role of Chinese face in the perpetration of dating partner violence. Journal of Interpersonal Violence, 27, 793-811. https:// doi.org/10.1177/0886260511423242

Children's Safety Network. (2012). Teen Dating Violence as a Public Health Issue. https://www. childrenssafetynetwork.org/sites/childrenssafetynetwork.org/files/TeenDatingViolenceasaPublicHealthIssue.pdf

Cohen, J., Shorey, R., Menon, S. y Temple, J. (2018). Predicting Teen Dating Violence Perpetration. Pediatrics, 141(4), e20172790. https:// doi.org/10.1542/peds.2017-2790

Cornelius, T. L y Resseguie, N. (2007). Primary and secondary prevention programs for dating violence: A review of the literature. Aggression and Violent Behavior, 12(3), 364-375. https:// doi.org/10.1016/j.avb.2006.09.006

Cutbush, S., Williams, J., Miller, S., Gibbs, D. y Clinton-Sherrod, M. (2018). Longitudinal Patterns of Electronic Teen Dating
Violence Among Middle School Students. Journal of Interpersonal Violence. https://oi. org/10.1177/0886260518758326

Diener, E., Emmons, R. A., Larsen, R. J. y Griffin, S. (1985). The Satisfaction with Life Scale. Journal of Personality Assessment, 49(1), 71-75. https:// doi.org/10.1207/s15327752jpa4901_13

Draucker, C. y Martsolf, D. S. (2010). The role of electronic communication technology in adolescent dating violence. Journal of Child and Adolescent Psychiatric Nursing, 23(3), 133-142. https://doi.org/10.1111/j.17446171.2010.00235.x

Erdem, A. y Sahin, R. (2017). Undergraduates' Attitudes Toward Dating Violence: Its Relationship with Sexism and Narcissism. International Journal of Higher Education, 6(6), 91-105. https://doi.org/10.5430/ijhe.v6n6p91

Fernández, L., Rodríguez, L., Molleda, C. y Rodríguez, F. (2015). Relaciones de noviazgo en jóvenes. Maltrato y religión. Infancia, Juventud y Ley, 6, 44-50.

Fernández-González, L., O’Leary, K. D y Muñoz-Rivas, M. J. (2013). Age-Related Changes in Dating Aggression in Spanish High School Students. Journal of Interpersonal Violence, 29(6), 1132-1152. https://doi. org/10.1177/0886260513506057

Eaton, D. K., Davis, K. S., Barrios, L., Brener, N. D. y Noonan, R. K. (2007). Associations of Dating Violence Victimization with Lifetime Participation, Co-Occurrence, and Early Initiation of Risk BehaviorsAmong U.S. High School Students. Journal of Interpersonal Violence, 22(5), 585-602. https://doi. org/10.1177/0886260506298831

Garay, F., González, M., Carrasco, M. y Amor, P. (2017). The Prevalence of Dating Violence: A Systematic Review. Psychologist Papers, 38(2), 135-147. https://doi.org/10.23923/pap. psicol2017.2831

García, F. E., Wlodarczyk, A., Reyes, A., San Cristóbal, C. y Solar, C. (2014). Violencia en la pareja, apoyo social y bienestar psicológico en adultos jóvenes. Ajayu, 12(2), 246-265.

Gómez, F., Barrientos, J., Guzmán, M., Cárdenas, M. y Bahamondes, J. (2017). Violencia de 
pareja en hombres gay y mujeres lesbianas chilenos: Un estudio exploratorio. Interdisdisciplinaria, 34(1), 57-72. https://doi.org/10.16888/ interd.2017.34.1.4

Herranz Bellido, J. (2013). Violencia de género en población adolescente. Guía de orientación para la familia Diputación de Alicante. Unidad de Igualdad. http://hdl.handle.net/10045/61450

Hirschi, T. (1969). Causes of delinquency. Berbeley, CA: University of California Press.

Holmes, K. y Sher, L. (2013). Dating violence and suicidal behavior in adolescents. International Journal of Adolescent Medicine and Health, 25(3), 257-261. https://doi.org/10.1515/ijamh2013-0059

Lavoie, F., Robitaille, L. y Hébert, M. (2000). Teen Dating Relationships and Aggression: An Exploratory Study. Violence against Women, 6(1), 6-36. https://doi. org/10.1177/10778010022181688

Lee, M., Reese-Weber, M. y Kahn, J. H. (2014). Exposure to family violence and attachment stylesaspredictors ofdating violence perpetration among men and women: A mediational model. Journal of Interpersonal Violence, 29(1), 20-43. https://doi.org/10.1177/0886260513504644

Liu, W., Mumford, E. A y Taylor, B. G. (2018). The Relationship Between Parents' Intimate Partner Victimization and Youths' Adolescent Relationship Abuse. Journal of Youth and Adolescence, 47(2), 321-333. https://doi.org/10.1007/s10964017-0733-1

Livingston, J. A., Eiden, R. D., Lessard, J., Casey, M., Henie, J. y Leonard, K. E. (2018). Etiology of Teen Dating Violence among Adolescent Children of Alcoholics. Journal of Youth and Adolescence, 47(3), 515-533. https://doi. org/10.1007/s10964-017-0730-4

Martine, H., Martin, B. y Francine, L. (2017). Prevalence of teen dating victimization among a representative sample of high school students in Quebec. International Journal of Clinical and Health Psychology, 17, 225-233. https://doi. org/10.1016/j.ijchp.2017.06

Martínez-Pecino, R. y Durán, M. (2016). I Love You but I Cyberbully You: The Role of Hostile Sexism. Journal of Interper- sonal Violence, 34(4), 812-825. https://doi. org/10.1177/0886260516645817

Miller, D. I., Eagly, A. H. y Linn, M. C. (2015). Women's representation in Science predicts national Gender-Science stereotypes: Evidence from 66 nations. Journal of Educational Psychology, 107(3), 631-644. https://doi. org/10.1037/edu0000005

Mitchell, C. (2019). Effect of religión on domestic violence perpetration among American adults. Theses 348, University of Missouri, ST, Louis. https://irl.umsl.edu/thesis/348

Montolío, C., Ros, C. y Portela, C. (2016). Programa para el contexto escolar de prevención de violencia en parejas adolescentes. Educar, 52(1), 11-31. https://doi.org/10.5565/ rev/educar.673

Mueller, V., Jouriles, E. N., McDonald, R. y Rosenfield, D. (2013). Adolescent beliefs about the acceptability of dating violence: Does violent behavior change them? Journal of Interpersonal Violence, 28(2), 436-450. https://doi. org/10.1177/0886260512454716

Murphy, M. C. y O’Leary, K. D. (1989). Psychological aggression predicts physical aggression in early marriage. Journal of Consulting and Clinical Psychology, 57(5), 579-582.

Observatorio del Pluralismo Religioso en España (2013). II Encuesta sobre opiniones y actitudes de los españoles ante la dimensión cotidiana de la religiosidad y su gestión pública. http:// www.observatorioreligion.es/upload/97/31/ II_Encuesta_sobre_opiniones_y_actitudes_de los_espanoles_ante_la_dimension_cotidiana de_la_religiosidad_y_su_gestion_publica.pdf.

Offenhauer, P. y Buchalter, A. (2011). Teen Dating Violence: A Literature Review and Annotated Bibliography. Library of Congress, Federal Research Division.

O'Leary, K. D., Malone, J. y Tyree, K. (1994). Physical aggression in early marriage: Prerelationship and relationship effects. Journal of Consulting and Clinical Psychology, 62(3), 594-602. https://doi.org/10.1037/0022006x.62.3.594

Parker, E. M., Johnson, S. L., Debnam, K. J., Milam, A. J. y Bradshaw, C. P. (2017). Teen 
Dating Violence Victimization Among High School Students: A Multilevel Analysis of School-Level Risk Factors. Journal of School Health, 87(9), 696-704. https://doi.org/10.1111/ josh.12538

Pedrosa, I., Juarros, J., Robles, A., Basteiro, J. y García-Cueto, E. (2015). Pruebas de bondad de ajuste en distribuciones simétricas, ¿qué estadístico utilizar? Universitas Psychologica, 14(1), 245-254. https://doi.org/10.11144/Javeriana. upsy13-5.pbad

Penado, M. y Rodicio-García, M. L. (2017). Analysis of self-concept in victims of gender based violence among adolescents. Suma Psicologica, 24(2), 107-114. https://doi.org/10.1016/j. sumpsi.2017.08.001

Perles, F., San Martin, J. y Canto, J. M. (2019). Gender and conflict resolutions strategies in Spanish teen couples: Their relationship with jealousy and emotional dependency. Journal of Interpersonal Violence, 34(7), 1461-1486. https://doi.org/10.1177/0886260516651316

Picard, P. (2007). Tech abuse in teen relationships. Chicago, IL: Teen Research Unlimited. http://www.loveisrespect.org/wp-content/ uploads/2009/03/liz-claiborne-2007-tech-relationship-abuse.pdf

Ponce-Díaz, C. R., Aiquina, J. J. y Arboccó, M. (2019). Dependencia emocional, satisfacción con la vida y violencia de pareja en estudiantes universitarias. Propósitos y Representaciones, 7(SPE), e351. https://doi.org/10.20511/pyr2019. v7nSPE. 351

Reidy, D., Holland, K. M., Cortina, K., Ball, B. Y Rosenbluth, B. (2017). Evaluation of the expect respect support group program: A violence prevention strategy for youth exposed to violence. Preventive Medicine. 100, 235-242. https://doi.org/10.1016/j.ypmed.2017.05.003

Reidy, D., Kearns, M., Houry, D., Valle, L., Holland, K. y Marshall, K. (2016). Dating Violence and Injury Among Youth Exposed to Violence. American Academy of Pediatrics, 137(2). https:// www.ncbi.nlm.nih.gov/pubmed/26826215

Rey-Anacona, C. (2009). Maltrato de tipo físico, psicológico, emocional, sexual y económico en el noviazgo: Un estudio exploratorio. Acta
Colombiana de Psicología, 12(2), 27-36.

Rivas, M., Roldan, P., Gimeno, M. y Díaz, A. (2015). Violencia de pareja online y offline en la adolescencia: El rol de la soledad y del género. Revista sobre la Infancia y la Adolescencia, 9, 85-97. https://doi.org/10.4995/reinad.2015.3898

Rodríguez Cabrera, A., Ramos, G. S., Contreras, M. E.y Cáceres, B. P. (2013). Estrategia educativa sobre promoción en salud sexual y reproductiva para adolescentes y jóvenes universitarios. Revista Cubana de Salud Pública, 39(1), 161-174.

Rodríguez-Fernández, A., Ramos-Díaz, E., Ros, I., Fernández-Zabala, A. y Revuelta, L. (2016). Bienestar subjetivo en la adolescencia: el papel de la resiliencia, el autoconcepto y el apoyo social percibido. Suma Psicológica, 23, 60-69. https://doi.org/10.1016/j.sumpsi.2016.02.002

Rodríguez, Y., Lameiras, M., Carrera, M. V. y Faílde, J. M. (2010). Evaluación de las actitudes sexistas en estudiantes españoles/as de Educación Secundaria Obligatoria. Psychologia: Avances de la Disciplina, 4(1), 11-24.

Roscoe, B. y Bensake, N. (1985). Courtship violence experienced by abused wives: Similiarities in Patterns of abuse. Family Relations, 34, 419-424. https://doi.org/10.2307/583582

Rouyer, V., Mieyaa, Y. y le Blanc, A. (2014). Gender socialization and the construction of gender identities. Revue Française de Pédagogie, 2(187), 97-137. https://doi.org/10.4000/ rfp.4494

Rubio-Garay, F., Carrasco, M. A., Amor, P. J. y López-González, M. A. (2015). Factores asociados a la violencia en el noviazgo entre adolescentes: una revisión crítica. Anuario de Psicología Jurídica, 25, 47-56. https://doi. org/10.1016/j.apj.2015.01.001

Saldivia, C. y Vizcarra, B. (2012). Consumo de Drogas y Violencia en el Noviazgo en Estudiantes Universitarios del Sur de Chile. Terapia Psicológica, 30(2), 43-49. https://doi. org/10.4067/S0718-48082012000200004

Sanabria, J. P., Jiménez Sierra, J., Parra, K. y Tordecilla, M. A. (2016). Influencia de la religión en la toma de decisiones sobre sexualidad en estudiantes católicos practicantes. Diversitas: Pers- 
pectivas en Psicología, 12(2), 231-242. https:// doi.org/10.15332/s1794-9998.2016.0002.05

Sánchez, V., Ortega, F. J., Ortega, R. y Viejo, C. (2008). Las relaciones sentimentales en la adolescencia: satisfacción, conflictos y violencia. Escritos de Psicología, 2(1), 97-109.

Santos, M. (2017). Sexismo ambivalente en la adolescencia y su relación con la autoestima (Trabajo de fin de Master). Universidad de Sevilla, España.

Sebastián, J., Verdugo, A. y Ortiz, B. (2014). Jealousy and Violence in Dating Relationships: Gender-Related Differences among a Spanish Sample. Spanish Journal of Psychology, 17, e94, 1-12. https://doi.org/10.1017/sjp.2014.99

Shaffer, C. M., Corona, R., Sullivan, T. N., Fuentes, V. y McDonald, S. E. (2018). Barriers and supports to dating violence communication between Latin adolescents and their mothers:

A qualitative analysys. Journal of Family Violence, 33, 133-145. https://doi.org/10.1007/ s10896-017-9936-1

Shorey, R. C., Wymbs, B., Torres, L., Cohen, J. R., Fite, P. J. y Temple, J. R. (2017). Does change in perceptions of peer teen dating violence predict change in teen dating violence perpetration over time? Aggressive Behavior, 44(2), 156-164. https://doi.org/10.1002/ab.21739

Smith, K., Cénat, J. M., Lapierre, A., Dion, J., Hébert, M y Côté, K. (2018). Cyber Dating Violence: Prevalence and Correlates Among High School Students from Small Urban Areas in Quebec. Journal of Affective Disorders. 234, 220-223. https://doi.org/10.1016/j. jad.2018.02.043

Smith, P. H., White, J. W. y Holland, L. J. (2003). A longitudinal perspective on dating violence among adolescent and college-age women.
American Journal of Public Health, 93(7), 11041109. https://doi.org/10.2105/ajph.93.7.1104

Temple, J. R., Choi, H. J., Reuter, T., Wolfe, D., Taylor C. A., Madigan, S. y Scott, L. E. (2018). Childhood Corporal Punishment and Future Perpetration of Physical Dating Violence. The Journal of Pediatrics, 194, 233-237. https://doi. org/10.1016/j.jpeds.2017.10.028

Valdivia, M. y González Bravo, L. A. (2014). Violencia en el noviazgo y pololeo: Una actualización proyectada hacia la adolescencia. Revista de Psicología (PUCP), 32(2), 329-355.

Vermeer, P. (2014). Religion and family life: An overview of current research and suggestions for future research. Religions, 5, 402-421. https://doi.org/10.3390/rel5020402

Wendt, S. y Zannettino, L. (2015). Domestic violence in diverse contexts: A re-examination of gender. New York: Routledge.

Viejo, C. (2012). Dating violence y cortejo adolescente. Un estudio sobre la violencia en las parejas sentimentales de los jóvenes andaluces. Córdoba, España: Servicio de Publicaciones de la Universidad de Córdoba.

Yan, F. A., Howard, D. E., Beck, K. H., Shattuck, T. y Hallmark-Kerr, M. (2009). Psychosocial correlates of physical dating violence victimization among latin early adolescents. Journal of Interpersonal Violence, 39, 119e1-119e8. https://doi.org/10.1177/0886260509336958

Yugueros, A. (2012). Consideraciones sobre la violencia hacia las mujeres. Socio-psicológica, 10, 37-110.

Zweig, J. M., Lachman, P., Yahner, J. y Dank, M. (2014). Correlates of cyber dating abuse among teens. Journal of Youth and Adolescence, 43, 1306-1321. https://doi.org/10.1007/s10964013-0047-x.

Recibido: 24 de abril de 2020 Aceptado: 19 de agosto de 2021 